\title{
Temporo-parietal junction as a mediator of reactive social behavior: the role of agreeableness
}

\author{
E. Merkulova*, A. Savostyanov, A. Bocharov, G. Knyazev \\ Laboratory of Psychophysiology of Individual Differences, Institute of Physiology and Basic Medicine, \\ Novosibirsk, Russia \\ *e-mail:merkaterine@gmail.com
}

Key words: social interactions; agreeableness; multilevel mediation analysis; reactive behavior; EEG

Motivation and Aim: Human behavior in social situations has two distinct components reactive and nonreactive. Reactive behavior is largely driven by the perceived behavioral manifestation of the opponent and is indispensable for successful social interactions. The ability and willingness to pay attention and correctly interpret verbal and nonverbal signs from the opponent and to tune his or her own behavior accordingly should depend on personality, but little is known about brain underpinning of this behavior and the impact of personality. In this study, we used a model of virtual social interactions, which allows to manipulate the emotional display of the 'opponent' and to register actor's responses to these manipulations.

Methods and Algorithms: High-density EEG was recorded throughout the experiment and participants additionally filled in the IPIP Big-Five Factor Markers [1]. The emotional stimulus category was used as a predictor, behavioral response as outcome, and sourcelevel event-related oscillatory power was used as a mediator in the multilevel mediation analysis [2].

Results: Significant mediation effects were revealed only in the theta frequency band. For the reactive response, a positive mediation effect was found in the Brodmann area 39. Event-related theta activity in this cluster increases as stimuli characteristics change from happy to neutral and from neutral to angry facial expressions and this increase is associated with increased probability of a more-aggressive/less-friendly behavioral response. Fisher z-transformed mediation coefficients (path AB) correlated with agreeableness $(r=0.50, p=0.001)$, but not with other personality variables. For the nonreactive response, a positive mediation effect effect did not correlate with personality variables.

Conclusion: The association between the predictor and the outcome was mediated by event-related theta activity in the right temporo-parietal junction and the strength of mediation was moderately positively associated with agreeableness, suggesting that the brain mechanism underlying reactive social behavior is more active in agreeable individuals.

Acknowledgements: Supported by the Russian Science Foundation (RSF) under Grant No. 17-18-01019.

\section{References}

1. Knyazev G.G., Mitrofanova L.G., Bocharov V.A. (2010) Validization of Russian version of Goldberg's "Big-Five factor markers" inventory. Psikhologicheskii Zhurnal. 31:100-110 (in Russian).

2. Wager T.D., Davidson M.L., Hughes B.L., Lindquist M.A., Ochsner K.N. (2008) Prefrontal-subcortical pathways mediating successful emotion regulation. Neuron. 59:1037-1050. 